\title{
A Perspective on the Impact of the Shine-Through Artifact in Clinical Applications of PET/MR Imaging
}

\author{
Osman Ratib \\ Division of Nuclear Medicine and Molecular Imaging, Department of Medical Imaging and Information Sciences, University Hospital \\ of Geneva, Geneva, Switzerland
}

$\mathbf{H}$ clinical practice, with the major vendors now providing hybrid scanners that are certified for clinical use. This new hybrid technique is certainly more complex and challenging than its predecessor, PET/CT (1). Beyond the technical challenge of the magnetic field interfering with PET detectors, which led to the development of a new generation of solid-state detectors that are MR-compatible, several other technical challenges still exist. The most widely discussed is calculation of tissue attenuation from MR images to correct PET images and accurately visualize and measure the true tracer concentrations in different parts of the body.

The current technique requires segmentation of anatomic structures to assign to soft tissue an estimated tissue-correction factor different from that assigned to air-filled cavities and lungs. Although an approximated correction such as this is sufficient for most clinical applications, its inaccuracy can limit the use of tracer concentration measurement for more precise studies of certain biomarkers.

The major limitation of this technique is the difficulty in accurately identifying bone structures on conventional MR images

\section{See page 951}

and thereby estimating the additional attenuation factor of bones (2). This limitation results in inaccuracy in the quantitative measurement of tracer concentration (such as standardized uptake value) in different organs, particularly those near dense bones. Although recent publications have shown that measurement of standardized uptake value may be inaccurate because of the lack of bone attenuation, the marginal range of error $(10 \%-$ $20 \%$ ) may not have a significant impact in most clinical applications. Awareness of such inaccuracy is being factored into the expert interpretation of imaging data and has only a limited effect on global diagnostic accuracy and patient management. Efforts are under way, however, to develop innovative techniques to identify bone structures on MR images so that the accuracy of

Received Mar. 13, 2015; revision accepted Mar. 16, 2015.

For correspondence or reprints contact: Osman Ratib, Hôpitaux Universitaires de Genève, Rue Gabrielle-Perret-Gentil, 4, 1211 Geneve 14, Suisse.

E-mail: osman.ratib@hcuge.ch

Published online Apr. 9, 2015.

COPYRIGHT (C) 2015 by the Society of Nuclear Medicine and Molecular Imaging, Inc.

DOI: $10.2967 /$ jnumed.115.155135 quantitative measurements of tracer concentrations can be improved (3).

Several other limitations of PET/MR in comparison with PET/ CT have also been identified and discussed in recent clinical validation studies. The most prominent is the limitation of MR in imaging lung tissue, therefore affecting the accuracy of diagnosing lung lesions. It is not uncommon that a patient with suspected lung lesions on PET/MR will then require CT or PET/CT of the lungs. Major artifacts from metallic implants are another limitation, as these are much more prominent on PET/MR than on PET/CT images and can seriously limit image interpretation.

One artifact that has not yet been clearly evaluated is the effect of the high magnetic field on the trajectory of positrons emitted by PET radioisotopes. In this issue of The Journal of Nuclear Medicine, Kolb et al. (4) report on this artifact. Many authors have predicted that the high magnetic field will reduce the positron range in the plane perpendicular to the static field, thus improving transaxial resolution. However, little has been mentioned about the possibility that axial resolution may be affected by the elongation of the positron range distribution along the magnetic field. Furthermore, depending on the energy of the positron and the type of tissue encountered, this phenomenon may generate a shine-through artifact in neighboring anatomic structures. This effect is nicely demonstrated in the paper by Kolb et al., with exquisite illustrations of its visual representation in animal models.

The work of Kolb et al. shows the risk of false interpretation of tracer uptake in tissue adjacent to a focus of high tracer uptake. This effect was previously observed by Abduhl-Fatah et al. in PET/CT images with high-energy PET isotopes $\left({ }^{124} \mathrm{I}\right)$ but was never observed with the lower-energy isotopes that are commonly used in clinical practice. In PET/MR scanners, this shine-through effect is exacerbated with lower-energy isotopes by the elongation of the positron range in the axial direction of the magnetic field. This phenomenon is observed only if the source of high tracer uptake is adjacent to an air cavity, such as in the animal model used by Kolb et al., in which the shine-through artifact was shown at the opposite side of the airway along the magnetic field. In denser tissues or even in the lungs, this phenomenon is much less prominent or even nonexistent. However, it should be acknowledged and well understood in areas near airways, particularly in head-andneck studies, for which PET/MR is preferred for assessment and follow-up of tumors (5).

The high metabolic activity of a tumor near airway cavities may generate a shine-through artifact in the opposite wall in the axial direction. Interpreting physicians must be trained to identify these 
artifacts and avoid misinterpretation of abnormal tracer uptake in normal tissue. When there is doubt, a complementary PET/CT acquisition of the same region may help eliminate the artifact (6), as nicely demonstrated by the comparison of PET/CT images with PET/MR images in the paper by Kolb et al. As they point out, it is fundamental to identify situations in which positron shine-through may hamper accurate quantification and diagnosis. This observation highlights the importance of proper training and in-depth understanding of the physics of PET/MR when studies are interpreted in clinical routine. Although such a phenomenon may not be frequent and may be easily identified, there is still a risk of erroneous interpretation of abnormal tracer uptake in areas of normal tissue.

\section{DISCLOSURE}

No potential conflict of interest relevant to this article was reported.

\section{REFERENCES}

1. Beyer T, Moser E. MR/PET or PET/MRI: does it matter? MAGMA. 2013;26: $1-4$.

2. Aznar MC, Sersar R, Saabye J, et al. Whole-body PET/MRI: the effect of bone attenuation during MR-based attenuation correction in oncology imaging. Eur $J$ Radiol. 2014;83:1177-1183.

3. Burger IA, Wurnig MC, Becker AS, et al. Hybrid PET/MR imaging: an algorithm to reduce metal artifacts from dental implants in Dixonbased attenuation map generation using a multiacquisition variableresonance image combination sequence. $J$ Nucl Med. 2015;56: 93-97.

4. Kolb A, Sauter AW, Eriksson L, et al. Shine-through in PET/MR imaging: effects of the magnetic field on positron range and subsequent image artifacts. J Nucl Med. 2015;56:951-954.

5. Varoquaux A, Rager O, Poncet A, et al. Detection and quantification of focal uptake in head and neck tumours: ${ }^{18}$ F-FDG PET/MR versus PET/CT. Eur J Nucl Med Mol Imaging. 2014;41:462-475.

6. Purohit BS, Ailianou A, Dulguerov N, Becker CD, Ratib O, Becker M. FDG-PET/ CT pitfalls in oncological head and neck imaging. Insights Imaging. 2014;5: 585-602. 